\title{
Microscopic surface structure of liquid alkali metals
}

\author{
H. Tostmann \\ Division of Applied Sciences and Department of Physics, Harvard University, Cambridge, Massachusetts 02138 \\ E. DiMasi \\ Department of Physics, Brookhaven National Laboratory, Upton, New York 11973-5000 \\ P. S. Pershan \\ Division of Applied Sciences and Department of Physics, Harvard University, Cambridge, Massachusetts 02138 \\ B. M. Ocko \\ Department of Physics, Brookhaven National Laboratory, Upton, New York 11973-5000 \\ O. G. Shpyrko \\ Division of Applied Sciences and Department of Physics, Harvard University, Cambridge, Massachusetts 02138 \\ M. Deutsch \\ Department of Physics, Bar-Ilan University, Ramat-Gan 52100, Israel
}

(Received 13 October 1999)

\begin{abstract}
We report an x-ray scattering study of the microscopic structure of the surface of a liquid alkali metal. The bulk liquid structure factor of the eutectic $\mathrm{K}_{67} \mathrm{Na}_{33}$ alloy is characteristic of an ideal mixture, and so shares the properties of an elemental liquid alkali metal. Analysis of off-specular diffuse scattering and specular $\mathrm{x}$-ray reflectivity shows that the surface roughness of the K-Na alloy follows simple capillary wave behavior with a surface structure factor indicative of surface-induced layering. Comparison of the low-angle tail of the $\mathrm{K}_{67} \mathrm{Na}_{33}$ surface structure factor with the one measured for liquid Ga and In previously suggests that layering is less pronounced in alkali metals. Controlled exposure of the liquid to $\mathrm{H}_{2}$ and $\mathrm{O}_{2}$ gas does not affect the surface structure, indicating that oxide and hydride are not stable at the liquid surface under these experimental conditions.
\end{abstract}

The structure of the free surface of a liquid metal (LM) is fundamentally different from that of a dielectric liquid, due to the strong coupling between conduction electrons and ion cores. ${ }^{1}$ At the liquid-vapor interface of a LM, the Coulomb interaction between the free electron Fermi gas and the classical gas of charged ions acts like an effective hard wall and forces the ions into ordered layers parallel to the surface. The existence of surface-induced layering in LM's has been verified unambiguously by experiment for liquid $\mathrm{Hg}^{2} \mathrm{Ga}^{3}$ and In. ${ }^{4}$ Layering is also present in liquid binary alloys, even though it may be suppressed by surface segregation ${ }^{5}$ or the formation of surface phases. , $^{6}$

Even though surface layering appears to occur in LM's in general, comparison of the detailed surface structure reveals qualitative differences between different LM's. ${ }^{4,8}$ In fact, very little is understood about how the surface-induced layering predicted for an ideal LM is affected by the details of the more complicated electronic structure exhibited by the polyvalent metals studied so far. One important goal therefore is to study the surface structure of those LM's that show the most ideal electronic structure in the bulk phase, characterized by itinerant conduction electrons which are only weakly perturbed by a small ionic pseudopotential. These LM's are referred to as nearly-free-electron type liquid metals. ${ }^{9}$ In a previous study we have compared the surface structure of liquid In, which is considered to be a nearly-free- electron type liquid metal, with the surface structure of liquid $\mathrm{Ga}$, which displays a considerable degree of covalency in the bulk. $^{4}$ However, even though liquid In has less tendency toward covalent bonding than $\mathrm{Ga}$, it is trivalent, and the most simple models do not adequately describe its electronic structure or metallic properties. ${ }^{11}$ The liquid metals closest to nearly-free-electron behavior are the monovalent alkali metals, which have a half-filled $s$ band and an almost spherical Fermi surface. ${ }^{10}$ The metallic properties of alkali metals are explained by the most simple solid state models such as the Drude theory. ${ }^{11}$ The facts that the conduction band is primarily formed from $s$-electron states and that the electron-ion interaction can be modeled by a weak pseudopotential have led to numerous theoretical studies on the electronic structure. At the free LM surface, virtually all theoretical studies and computer simulations have been applied to liquid $\mathrm{Na}, \mathrm{K}$, and Cs. ${ }^{1,12}$

A particularly interesting question concerns the possible correlation between surface tension and surface structure. In general, LM's have surface tensions that are at least an order of magnitude higher than the surface tensions of all other types of liquids. ${ }^{7}$ Liquid alkali metals are the only exception to this pattern. For example, $\mathrm{Ga}$ and $\mathrm{Cs}$ melt at about the same temperature but the surface tension of $\mathrm{Ga}$ is greater than that of Cs by about an order of magnitude. If the high surface tension of liquid metals is related to surface-induced 


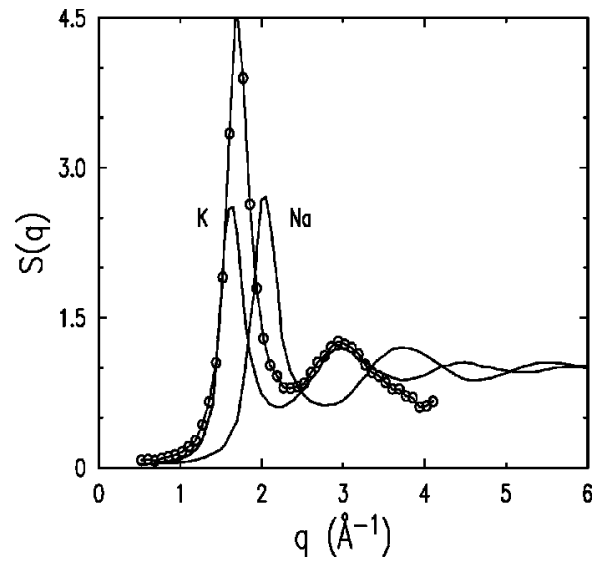

FIG. 1. (O) Static structure factor of liquid $\mathrm{K}_{67} \mathrm{Na}_{33}$ as a function of the in-plane momentum transfer. (-) bulk structure factors of pure liquid $\mathrm{K}$ and $\mathrm{Na}$ from Ref. 14.

layering, it would be reasonable to expect that liquid alkali metals should display weak or no layering at the surface. This is contradicted by computer simulations showing pronounced layering for liquid Cs. ${ }^{1}$

The alkali alloy $\mathrm{K}_{67} \mathrm{Na}_{33}$ is more suitable experimentally for such a study than are the elemental liquid alkali metals. Among these, Li deviates from nearly-free electron type behavior, ${ }^{11,13}$ while the high equilibrium vapor pressures of the other elemental alkali metals prevent the use of an ultrahigh vacuum (UHV) environment over a long period of time without noticeable evaporation of material. Experiments under UHV conditions are mandated by the high reactivity of liquid alkali metals toward water and oxygen. ${ }^{13}$ When liquid $\mathrm{K}$ and $\mathrm{Na}$ are alloyed in the eutectic composition (67 at. \% $\mathrm{K}$ ), however, a vapor pressure well below $10^{-10}$ Torr is obtained. The low melting point of this alloy $\left(-12.6^{\circ} \mathrm{C}\right)$ is an additional advantage, since the thermal surface roughness is considerably reduced. ${ }^{4}$ This binary alloy appears to be well suited for emulation of the properties of an elemental liquid alkali metal. The total static structure factor $S(q)$ of bulk liquid $\mathrm{K}_{67} \mathrm{Na}_{33}$, shown in Fig. 1, is characteristic of random mixing, without subpeak or asymmetry of the first peak. ${ }^{14}$ The first peak of $S(q)$ for the alloy lies between those of pure $\mathrm{K}$ and $\mathrm{Na}$, as expected for an ideal mixture. ${ }^{14}$ More importantly, the electronic structure of the homovalent K-Na alloys is as nearly-free-electron-like as the electronic structure of the pure components. ${ }^{15}$ Even if there were some homocoordination or surface enrichment of $\mathrm{K}$ driven by the lower surface tension of $\mathrm{K}$ as compared to $\mathrm{Na}$, it would not be discernible in our structural measurements, since $\mathrm{x}$-ray scattering is sensitive to the distribution of the electron density, which is virtually identical for $\mathrm{K}$ and $\mathrm{Na}\left(0.25 e / \AA^{3}\right.$ and $0.27 e / \AA^{3}$, respectively).

The eutectic alloy was prepared by mixing liquid $\mathrm{K}$ and $\mathrm{Na}$ in an inert atmosphere glove box $\left(<2 \mathrm{ppm} \mathrm{H}_{2} \mathrm{O}\right.$, $<1 \mathrm{ppm} \mathrm{O}_{2}$ ). The alloy was transferred into a stainless steel reservoir and sealed with a Teflon $\mathrm{O}$ ring. The design of the reservoir is similar to the one used in recent $\mathrm{Hg}$ experiments. ${ }^{8}$ The reservoir is connected to a stainless steel UHV valve which is attached to a UHV flange. This assembly was connected to the UHV chamber, which was baked out at $150^{\circ} \mathrm{C}$ until a vacuum in the $10^{-10}$ Torr range resulted. After reaching this pressure, the Mo sample pan (20 $\mathrm{mm}$ diameter, $1 \mathrm{~mm}$ depth) in the UHV chamber was sputtered clean with $\mathrm{Ar}^{+}$ions. Sputtering removes the native Mo oxide layer and facilitates the wetting of the sample pan by the liquid alloy. After sputtering, the UHV valve to the reservoir was opened and the liquid alloy metered into the sample pan. As we will show below, our measurements under these conditions are consistent with a surface free of oxide on an atomic level.

Experiments were carried out using the liquid surface spectrometer at beamline X25 at the National Synchrotron Light Source, operating with an $\mathrm{x}$-ray wavelength $\lambda$ $=0.65 \AA$ and a resolution of $\Delta q_{z} \approx 0.05 \AA^{-1}$. In the x-ray reflectivity geometry, incident angle $\alpha$ and reflected angle $\beta=\alpha$ are varied simultaneously with $\beta$ detected within the plane of incidence. The background intensity, due mainly to scattering from the bulk liquid, is subtracted from the specular signal by displacing the detector out of the reflection plane by slightly more than one resolution width., ${ }^{4,5,16}$ The specular reflectivity from the surface, $R\left(q_{z}\right)$, is a function of the normal component, $q_{z}=(4 \pi / \lambda) \sin \alpha$, of the momentum transfer $\vec{k}_{\text {out }}-\vec{k}_{\text {in }}=\left(0,0, q_{z}\right) . \quad R\left(q_{z}\right)$ yields information about surface roughness and surface-normal structure and may be approximated as ${ }^{4}$

$$
R\left(q_{z}\right)=R_{f}\left(q_{z}\right)\left|\Phi\left(q_{z}\right)\right|^{2}\left(\frac{q_{\text {res }}}{q_{\max }}\right)^{\eta}
$$

where $R_{f}\left(q_{z}\right)$ is the Fresnel reflectivity of a flat, laterally homogeneous surface. The surface structure factor $\Phi\left(q_{z}\right)$ is the Fourier transform of the gradient of the intrinsic surfacenormal density profile. The power-law term with $\eta$ $=\left(k_{B} T / 2 \pi \gamma\right) q_{z}^{2}$ accounts for roughening of the intrinsic density profile by capillary waves (CW's). Scattering of $\mathrm{x}$-ray photons by CW's outside the detector resolution $q_{\text {res }}$ results in a loss in observed intensity. The short-wavelength cutoff $q_{\max }$ is determined by the particle size $a$ with $q_{\max }$ $\approx \pi / a$. The observed reflectivity is reduced when either the temperature $T$ is increased or the surface tension $\gamma$ is reduced.

For off-specular diffuse scattering, the incoming angle $\alpha$ is kept constant while $\beta$ is varied, straddling the specular condition $\alpha=\beta$. The bulk diffuse background has to be subtracted from the measured overall intensity. An accurate description of the theoretical diffuse scattering requires inclusion of the contribution of the surface background to the off-axis background. ${ }^{4,16}$ The line shape of the diffuse scattering is determined principally by the power-law term in Eq. (1). Analysis of diffuse scattering gives access to in-plane correlations of the surface and surface inhomogeneities that might be present. ${ }^{16}$ Simultaneous analysis of reflectivity and diffuse scattering allows for a consistent determination of surface roughness and structure. As has been pointed out recently, ${ }^{17}$ it is only for $\eta<2$ that the reflected intensity displays the cusplike singularity centered at the specular condition $\alpha=\beta$. For $\eta \geqslant 2$, it is not possible to distinguish surface scattering from bulk diffuse scattering since both are dominated by correlations of the same length scale. The value of $q_{z}$ at which $\eta$ reaches the limit of 2 at room temperature is about $1.7 \AA^{-1}$ for $\mathrm{K}_{67} \mathrm{Na}_{33}$.

Diffuse scattering measurements from the surface of $\mathrm{K}_{67} \mathrm{Na}_{33}$ taken at different incoming angles $\alpha$ [Fig. 2(a)] 

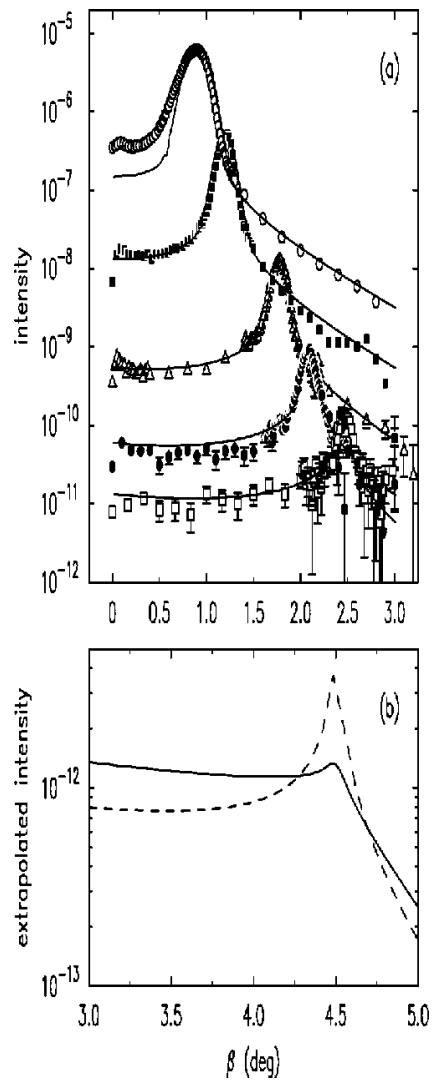

FIG. 2. (a) Off-specular diffuse scattering from the surface of liquid $\mathrm{K}_{67} \mathrm{Na}_{33}$ for different incoming angles $\alpha$ : (○) $0.9^{\circ}$ (multiplied by 25 for clarity), (ם) $1.2^{\circ}(\times 5),(\triangle) 1.77^{\circ}$, (○) $2.1^{\circ}(\times 0.2)$, and $(\square): 2.45^{\circ}(\times 0.1)$. (b) Extrapolated diffuse scattering (assuming no surface layering) for $\alpha=4.5^{\circ}$ : (一) resolution of the present experiment $\Delta q_{z} \approx 0.03 \AA^{-1}$. ( - - -) assuming the resolution attainable at a low-divergence $\mathrm{x}$-ray source, $\Delta q_{z} \approx 3 \times 10^{-4} \AA^{-1}$. (Intensity $\times 100$.)

show that the liquid alloy has a uniform surface roughened by capillary waves. The solid lines through the data are calculated from CW theory [power-law term in Eq. (1)] with the surface roughness entirely due to thermally activated surface waves. This model has no adjustable parameters, and the surface tension of $0.110 \pm 0.003 \mathrm{~N} / \mathrm{m}$ is consistent with macroscopic measurements. ${ }^{18}$ The agreement between the data and $\mathrm{CW}$ theory is excellent. The only limitation is in the low-angle region $\left(\alpha=0.9^{\circ}\right.$ and $\beta<\alpha$ ), where the footprint of the incoming beam is larger than the flat part of the sample. Apart from this deviation, the sample shows no excess scattering which would be expected from an inhomogeneous surface. ${ }^{4,16}$ The absence of excess diffuse scattering indicates that the alloy surface is free of microscopic patches or islands of possible contaminants such as oxide or hydride.

As a result of this excellent agreement between $\mathrm{CW}$ theory and the measured diffuse scattering, the reflectivity from the alloy surface shown in Fig. 3 (open squares) can be compared to the calculated reflectivity from a $\mathrm{K}_{67} \mathrm{Na}_{33}$ surface which is roughened by $\mathrm{CW}$ but displays no structure [i.e., $\Phi\left(q_{z}\right)=1$ ]. The dashed line in Fig. 3 displays this theoretical prediction and it is obvious that the data rise above it, consistent with the low-angle behavior of surface-induced layering observed previously. ${ }^{3,4}$ This point is emphasized further in the inset where the measured $R\left(q_{z}\right)$ is divided by

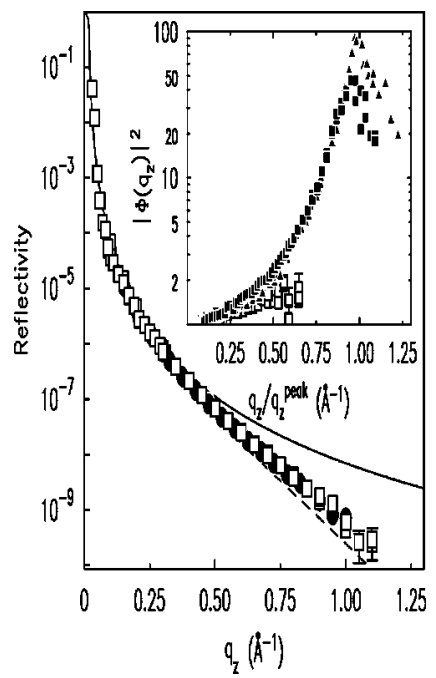

FIG. 3. X-ray reflectivity from a clean liquid $\mathrm{K}_{67} \mathrm{Na}_{33}$ surface at room temperature $(\square)$ and $\mathrm{K}_{67} \mathrm{Na}_{33}$ following an exposure to 3000 $\mathrm{L}$ of oxygen $(\mathbf{O})$ Also included for reference: $(-)$ calculated Fresnel reflectivity from an ideal $\mathrm{K}_{67} \mathrm{Na}_{33}$ surface; (- - -) calculated reflectivity $R_{\mathrm{cw}}$ from a $\mathrm{K}_{67} \mathrm{Na}_{33}$ surface roughened by capillary waves at room temperature. Inset: Surface structure factor $\left|\Phi\left(q_{z}\right)\right|^{2}$ of liquid $\mathrm{K}_{67} \mathrm{Na}_{33}$ at room temperature $(\square)$. For comparison the surface structure factors for liquid $\mathrm{Ga}$ (closed triangles) and In (closed boxes) are included. $q_{z}$ is normalized to the position of the first peak in the bulk $S(q)$.

$R_{f}$ and the power-law CW term in Eq. (1) to obtain a direct measure of the surface structure factor $\Phi\left(q_{z}\right)$. As can be seen, the value of $\Phi\left(q_{z}\right)$ rises above unity with increasing $q_{z}$, as expected when constructive interference of $\mathrm{x}$ rays due to surface layering is present. The low-angle tail of the surface structure factor of $\mathrm{K}_{67} \mathrm{Na}_{33}$ is now compared with that of liquid $\mathrm{Ga}$ and In reported previously. ${ }^{3,4}$ To normalize $q_{z}$ to the atomic size, $q_{z}$ has been divided by $q_{z}^{\max }$ of the first maximum of $S(q) .{ }^{19}$ For $\mathrm{K}_{67} \mathrm{Na}_{33}, \quad q_{z}^{\max } \approx 1.7 \AA^{-1}$ whereas for $\mathrm{Ga}$ and In $q_{z}^{\max } \approx 2.4 \AA^{-1}$. It is evident that the surface structure factor indicative of surface-normal layering does not increase as quickly for $\mathrm{K}_{67} \mathrm{Na}_{33}$ as for Ga or In. Assuming that the same layering model that successfully describes the surface of Ga (Ref. 3) and In (Ref. 4) also applies to $\mathrm{K}_{67} \mathrm{Na}_{33}$, the measurement indicates that either the surface layering of the alloy is significantly weaker than that of both Ga or In, or the length over which the layering decays into the bulk is significantly shorter. One can speculate that there might be a correlation between these layering length scales and the surface tension. This would mean that weak or quickly decaying layering is expected for LM's with exceptionally low surface tension. Clearly, data extending well beyond $q_{z}=1 \AA^{-1}$ are needed to support this conclusion.

Several factors contribute to the difficulty of extending reflectivity measurements to values of $q_{z}$ large enough to cover the expected position of the layering peak. The reflected intensity falls over almost ten orders of magnitude, while the background from the bulk structure factor increases, as this value of $q_{z}$ is approached. We must also consider that the exponent $\eta$ approaches its limiting value of 2 for $q_{z} \rightarrow 1.7 \AA^{-1}$ so that the specular cusp becomes broadened and ultimately lost in the diffuse background. Because of these effects, simply increasing the photon flux is not 
sufficient to improve the measurement substantially. A better approach is to significantly improve the resolution. This is illustrated in Fig. 2(b), where the calculated diffuse scattering for $\alpha=4.5^{\circ}$ (corresponding to $q_{z}=1.5 \AA^{-1}$ ) is shown for the current experimental resolution (solid line) and for a resolution sharper by a factor of 200 (dashed line). Although the intensity is much reduced in the latter case, the peak is much better defined. Reflectivity measurements conducted at low divergence synchrotron x-ray sources that are presently becoming available may make such extensions of the $q_{z}$ range possible.

To explore the effect of possible contaminants on the surface structure, we deliberately exposed the liquid alloy surface to controlled amounts of pure oxygen and hydrogen gas, measured in units of monolayer surface coverage (langmuir). ${ }^{20}$ The liquid alkali surface has been exposed to up to $3000 \mathrm{~L}$ oxygen and up to $6000 \mathrm{~L}$ hydrogen. As an example, $R\left(q_{z}\right)$ at the maximum exposure of $\mathrm{O}_{2}$ is shown as filled circles in Fig. 3. In no case was a measurable effect on $R\left(q_{z}\right)$ observed, indicating no change in the surface-normal structure. This insensitivity of the liquid $\mathrm{K}_{67} \mathrm{Na}_{33}$ surface to small amounts of oxygen is in striking contrast to similar experiments on liquid $\mathrm{Ga}$ (Ref. 20) and $\mathrm{Hg}$ (Ref. 21) where the formation of a thin homogeneous oxide film has been observed upon exposure of the liquid surface to as little as $200 \mathrm{~L}$ of oxygen. This difference is due to the fact that the low surface tension of liquid alkali metals precludes segregation of oxide from the bulk. By contrast, high surface tension LM's such as $\mathrm{Ga}$ or $\mathrm{Hg}$ lower their high overall surface energy by segregation of a contaminant film. These results are also in agreement with photoelectron spectroscopy experiments requiring a similarly clean liquid alkali metal surface. $^{22}$

In summary, we have shown that it is possible to investigate the surface structure of an atomically clean liquid alkali metal under UHV conditions. Exposing the sample to up to $6000 \mathrm{~L}$ of $\mathrm{H}_{2}$ or $\mathrm{O}_{2}$ has no effect on the atomic surface structure since the exceptionally low surface tension of alkali metals precludes contaminant segregation. From the diffuse scattering we extract the surface roughness, which agrees well with capillary wave theory, and with the macroscopic surface tension reported in the literature. The $q_{z}$ dependence of the surface structure factor determined from reflectivity is consistent with constructive interference of $\mathrm{x}$ rays due to surface-normal layering. This surface-induced layering in liquid $\mathrm{K}_{67} \mathrm{Na}_{33}$ appears to be much weaker than that found in high surface tension LM's such as In or Ga.

This work was supported by the U.S. DOE Grant No. DE-FG02-88-ER45379 and the U.S.-Israel Binational Science Foundation, Jerusalem. Brookhaven National Laboratory is supported by U.S. DOE Contract No. DE-AC0298CH10886.
${ }^{1}$ J. G. Harris, J. Gryko, and S. A. Rice, J. Chem. Phys. 87, 3069 (1987).

${ }^{2}$ O. M. Magnussen, B. M. Ocko, M. J. Regan, K. Penanen, P. S. Pershan, and M. Deutsch, Phys. Rev. Lett. 74, 4444 (1995).

${ }^{3}$ M. J. Regan, E. H. Kawamoto, S. Lee, P. S. Pershan, N. Maskil, M. Deutsch, O. M. Magnussen, B. M. Ocko, and L. E. Berman, Phys. Rev. Lett. 75, 2498 (1995).

${ }^{4}$ H. Tostmann, E. DiMasi, P. S. Pershan, B. M. Ocko, O. G. Shpyrko, and M. Deutsch, Phys. Rev. B 59, 783 (1999).

${ }^{5}$ M. J. Regan, P. S. Pershan, O. M. Magnussen, B. M. Ocko, M. Deutsch, and L. E. Berman, Phys. Rev. B 55, 15874 (1997).

${ }^{6}$ H. Tostmann, E. DiMasi, P. S. Pershan, B. M. Ocko, O. G. Shpyrko, and M. Deutsch, Ber. Bunsenges. Phys. Chem. 102, 1136 (1998).

${ }^{7}$ E. DiMasi and H. Tostmann, Synchrotron Radiat. News 12, 41 (1999).

${ }^{8}$ E. DiMasi, H. Tostmann, B. M. Ocko, P. S. Pershan, and M. Deutsch, Phys. Rev. B 58, 13419 (1998).

${ }^{9}$ J. M. Ziman, Philos. Mag. 6, 1013 (1961).

${ }^{10}$ N. H. March, Liquid Metals (Cambridge University Press, Cambridge, 1990)

${ }^{11}$ N. W. Ashcroft and N. D. Mermin, Solid State Physics (Saunders College Publishing, Orlando, FL, 1976).

${ }^{12}$ M. Hasegawa and M. Watabe, J. Phys. C 15, 353 (1982); E.
Chacon, F. Flores, and G. Navascues, J. Phys. F: Met. Phys. 14, 1587 (1984); M. A. Gomez and E. Chacon, Phys. Rev. B 49, 11 405 (1994).

${ }^{13}$ C. C. Addison, The Chemistry of the Liquid Alkali Metals (WileyInterscience, New York, 1984).

${ }^{14}$ Y. Waseda, The Structure of Non-Crystalline Materials (McGraw-Hill, New York, 1980).

${ }^{15}$ M. Shimoji, Liquid Metals (Academic Press, London, 1977), pp. 142-148.

${ }^{16}$ M. Fukuto, R. K. Heilmann, P. S. Pershan, J. A. Griffiths, S. M. Yu, and D. A. Tirrell, Phys. Rev. Lett. 81, 3455 (1998).

${ }^{17}$ P. S. Pershan, Synchrotron Radiat. 12, 10 (1999).

${ }^{18}$ V. K. Sheriev, T. P. Osiko, and O. G. Ashkhotov, High Temp. 29, 85 (1991).

${ }^{19}$ The position of the layering peak is expected to be close to that of the first peak of the bulk $S(q)$.

${ }^{20}$ M. J. Regan, H. Tostmann, P. S. Pershan, O. M. Magnussen, E. DiMasi, B. M. Ocko, and M. Deutsch, Phys. Rev. B 55, 10786 (1997).

${ }^{21}$ H. Tostmann, E. DiMasi, P. S. Pershan, B. M. Ocko, O. G. Shpyrko, and M. Deutsch, J. Non-Cryst. Solids 250-252, 1982 (1999).

${ }^{22}$ G. Indlekofer and P. Oelhafen, J. Non-Cryst. Solids 156-158, 226 (1993). 